\title{
Effect of Nonsurgical Periodontal Therapy on Serum Level of Interleukin-6 and Tumor Necrosis Factor- $\alpha$ in Chronic Periodontitis Patients with and without Hypothyroidism
}

\author{
Indhuja R Saraswathi ${ }^{1}$, Arun Sadasivan ${ }^{2}$, Elizabeth Koshi ${ }^{3}$, Steffi Vijayakumar ${ }^{4}$, Chitra G Vallabhan $^{5}$, Sheethel M Vrinda ${ }^{6}$
}

\begin{abstract}
Aim: The aim of this article was to determine the effect of nonsurgical periodontal therapy on clinical parameters and also to evaluate serum level of interleukin (IL)- 6 and tumor necrosis factor (TNF)- $\alpha$ in chronic periodontitis patients with and without hypothyroidism.

Materials and methods: Subjects were 40 female patients (20 systemically healthy subjects with chronic periodontitis and 20 subjects with hypothyroidism and chronic periodontitis). Clinical parameters like plaque score, gingival score, gingival recession, probing pocket depth, and clinical attachment level were recorded at baseline and 4 weeks after nonsurgical periodontal therapy. Nonsurgical periodontal therapy included oral hygiene instructions and full-mouth scaling and root planing. A total of $5 \mathrm{~mL}$ of venous blood sample was collected from each subject at baseline and 4 weeks after nonsurgical periodontal therapy.

Results: The clinical parameters and biochemical parameters like IL- 6 and TNF- $a$ in both groups showed pronounced reduction at the end of 4 weeks following nonsurgical periodontal therapy. However, simultaneous comparison of clinical and biochemical parameters between the two groups was not statistically significant.

Conclusion: This study provides evidence that nonsurgical periodontal therapy contribute to reduction in serum level of IL- 6 and TNF- $a$ in systemically healthy subjects with chronic periodontitis and subjects with hypothyroidism and chronic periodontitis.

Clinical significance: The prevalence of periodontitis as well as hypothyroidism is increasing nowadays. Interleukin- 6 and TNF- $a$ are found to play a significant role in pathobiology of both periodontitis and hypothyroidism. With the treatment of periodontitis, it is found that there is improvement in periodontal and thyroid status of the patients. So, it is an alert to health professionals about the relation of hypothyroidism and periodontitis so that they consider a multidisciplinary approach.
\end{abstract}

Keywords: Hypothyroidism, Interleukin-6, Periodontitis, Thyroid function test, Tumor necrosis factor-a.

The Journal of Contemporary Dental Practice (2020): 10.5005/jp-journals-10024-2812

\section{INTRODUCTION}

Periodontal diseases are bacterial infections characterized by inflammation and destruction of attachment apparatus, often leading to tooth loss. Although periodontal diseases are initiated by bacteria that colonize the tooth surface and gingival sulcus, that host response is believed to play an essential role in the breakdown of connective tissue and bone. ${ }^{1}$ The strongest type of causal relationship is the association of systemic inflammation and periodontal disease.

Thyroid is the principal endocrine gland that regulates body functions, growth, and metabolism. Various studies have estimated that around 42 million people suffer from thyroid diseases in India with a significantly higher prevalence in women. ${ }^{2}$

Thyroid gland is a bilobular structure composed of a right lobe and a left lobe connected by an isthmus. ${ }^{3}$ The major manifestations of thyroid dysfunction are hypothyroidism and hyperthyroidism. Hyperthyroidism is due to excessive thyroid hormone secretion. While hypothyroidism is due to deficient thyroid hormone secretion. ${ }^{4}$ Thyroid hormones play an important role in bone resorption by influencing osteoprotegerin and receptor activator of NF-KB ligand (RANKL) mechanisms. There is a positive association of thyroid hormone with markers of inflammation such as interleukin (IL)-1, IL-2, IL-6, natural killer T cells, memory helper T cells, tumor necrosis factor- $\alpha$ (TNF- $\alpha$ ), and transforming growth factor- $\beta .^{5}$

In periodontium, TNF- $a$ and IL- 6 seem to be an important inflammatory cytokines which act as a causative mediator of
${ }^{1-6}$ Department of Periodontics and Oral Implantology, Sree Mookambika Institute of Dental Sciences and Research, Tamil Nadu, India

Corresponding Author: Indhuja R Saraswathi, Department of Periodontics and Oral Implantology, Sree Mookambika Institute of Dental Sciences and Research, Tamil Nadu, India, Phone: +91 7708721743, e-mail: indhuravindran666@gmail.com

How to cite this article: Saraswathi IR, Sadasivan A, Koshi E, et al. Effect of Nonsurgical Periodontal Therapy on Serum Level of Interleukin-6 and Tumor Necrosis Factor- $a$ in Chronic Periodontitis Patients with and without Hypothyroidism. J Contemp Dent Pract 2020;21(4):410-415.

Source of support: Nil

Conflict of interest: None

periodontal tissue destruction. ${ }^{6}$ Interleukin- 6 and TNF-a are two major pro-inflammatory cytokines that are locally produced in different tissues in different pathological situations including thyroid dysfunction. ${ }^{7}$ These locally produced factors enter systemic circulation and spread in the whole body including into periodontium producing metalloproteinase molecules that mediate connective tissue destruction and induce the differentiation and activation of osteoclasts, leading to alveolar bone destruction. ${ }^{8}$ Thyroid dysfunction has a negative effect on IL- 6 and TNF- $a$, which play an important role in osteoclast differentiation and function

(C) The Author(s). 2020 Open Access This article is distributed under the terms of the Creative Commons Attribution 4.0 International License (https://creativecommons. org/licenses/by-nc/4.0/), which permits unrestricted use, distribution, and non-commercial reproduction in any medium, provided you give appropriate credit to the original author(s) and the source, provide a link to the Creative Commons license, and indicate if changes were made. The Creative Commons Public Domain Dedication waiver (http://creativecommons.org/publicdomain/zero/1.0/) applies to the data made available in this article, unless otherwise stated. 
without any influence from RANKL-receptor activator of NF-KB interaction. ${ }^{9}$ Bone resorption in hypothyroid patients is seen more when compared with hyperthyroid due to slower bone turnover rate causing retarded bone growth and maturation. ${ }^{10}$ Nonsurgical periodontal therapy has shown to reduce the oxidative stress within the periodontium and also reduce the inflammation, affecting the level of pro-inflammatory cytokines. ${ }^{11}$

In this study, we compare the effect of nonsurgical periodontal therapy on serum levels of IL- 6 and TNF-a in chronic periodontitis patients with and without thyroid dysfunction.

\section{Materials and Methods}

\section{Study Population}

This is a comparative interventional study for evaluating the levels of serum IL-6 and TNF- $a$ in chronic periodontitis patients with and without hypothyroidism before and after scaling and root planing procedure. The subjects for this study were selected from the outpatients of Department of Periodontics, Sree Mookambika Institute of Dental Sciences and Research and outpatients of Department of General Medicine and General Surgery of Sree Mookambika Institute of Medical Sciences (SMIMS), Kulasekharam. The study protocol was approved by the Ethical Committee of SMIMS, Kulasekharam, and was registered under the Clinical Trial Registry of India (CTRI/2019/10/021557). This study included recruitment of 40 subjects with chronic periodontitis. The selected patients were assigned into two groups, each group consisting of 20 female patients: 20 systemically healthy subjects with chronic periodontitis (control group) and 20 subjects with chronic periodontitis and hypothyroidism (study group). All patients were eligible for this study if they had been diagnosed with chronic periodontitis and showed attachment loss of $>$ $5 \mathrm{~mm}$ at more than $30 \%$ of the site, patients with bleeding on probing, patients with $>20$ functional teeth, and female patients between age group 18 years and 60 years, since the prevalence of hypothyroidism was significantly more in females than males. ${ }^{12}$ Serum concentration of thyroid-stimulating hormone (TSH), $\mathrm{T}_{3}$, and free tetraiodothyronine $\left(\mathrm{FT}_{4}\right)$ were analyzed for detection of thyroid dysfunction. According to American Association of Clinical Endocrinolgy, elevated TSH $(>5.5 \mu \mathrm{lU} / \mathrm{mL})$ and low circulating free $\mathrm{T}_{4}$ levels are indicative of hypothyroidism. ${ }^{13}$ Participants were excluded if they had a history of any systemic condition except hypothyroidism, smokers and former smokers, alcoholics and former alcoholics, obese, pregnant and lactating woman, subjects over 60 years of age, patients presenting any kind of inflammatory disease within last 3 months, and patients who had received professional periodontal treatment during the 6-month period prior to the study. The study protocol was explained, and a written informed consent was obtained from each participant.

\section{Periodontal Treatment}

Periodontal examination was conducted in the Department of Periodontics, Sree Mookambika Institute of Dental Sciences and Research, Kulasekharam. All investigations were performed by a single examiner. The following clinical parameters were assessed using William's graduated periodontal probe. Clinical parameters were assessed at six sites of all teeth (mesiobuccal, midbuccal, distobuccal, mesiolingual, midlingual, and distolingual) at baseline and 4 weeks after nonsurgical periodontal therapy: Plaque index (Silness and Loe 1964), Gingival index (Loe and Silness P 1963), clinical attachment level, and probing pocket depth. Initial periodontal therapy includes oral hygiene instructions, full-mouth scaling, and root planing. All patients received oral hygiene instructions and underwent full-mouth subgingival scaling and root planing that were performed under local anesthesia using Gracey curettes (Hu-Friedy, Chicago) at baseline. The periodontal parameters were recorded at 4 weeks following the nonsurgical periodontal therapy.

\section{Laboratory Assessments}

Under sterile conditions from each patient of both groups, $5 \mathrm{~mL}$ of venous blood sample was collected at baseline and 1 month after the nonsurgical periodontal therapy from the antecubital fossa venipuncture using the 23 -gauge needle with $5-\mathrm{mL}$ syringe (Fig. 1). Collected blood was placed in clot activator tube for 30 minutes at room temperature and centrifuged at 3,000 rpm for 10 minutes (Fig. 2).

The serum obtained was transferred to a plastic vial and stored at $-80^{\circ} \mathrm{C}$ until the time of an assay (Fig. 3). The amount of cytokine levels of IL- 6 and TNF- $a$ in the serum sample was determined using a commercially available enzyme-linked immunosorbent assay (ELISA) kit (Biowel-Diaclone, France) according to the protocol recommended by the manufacturer (Fig. 4). Biochemical evaluation of IL- 6 and TNF- $a$ was done at the molecular diagnostics wing at Rajiv Gandhi Center for Biotechnology, Trivandrum.

\section{Statistical Analysis}

The data were analyzed by Statistical Package for Social Sciences (SPSS 22.0) version. Paired $t$ test was applied to find the statistical significant before and after treatment within and between the groups. Analysis of variance (post hoc) followed by Dunnet $t$ test was applied to find a statistical significant between the groups. The value of $p<0.05$ was considered statistically significant at $95 \%$ confidence interval.

\section{Results}

Both group I and group II consisted of 40 female subjects between 18 and 60 years of age, where the maximum subjects fell under the category of 31 to 50 years (Table 1).

A significant $(p<0.05)$ reduction in mean periodontal parameters within the study group occurred at 4 weeks after nonsurgical periodontal therapy. Similarly, there was a statistically significant difference in the mean biochemical parameters at

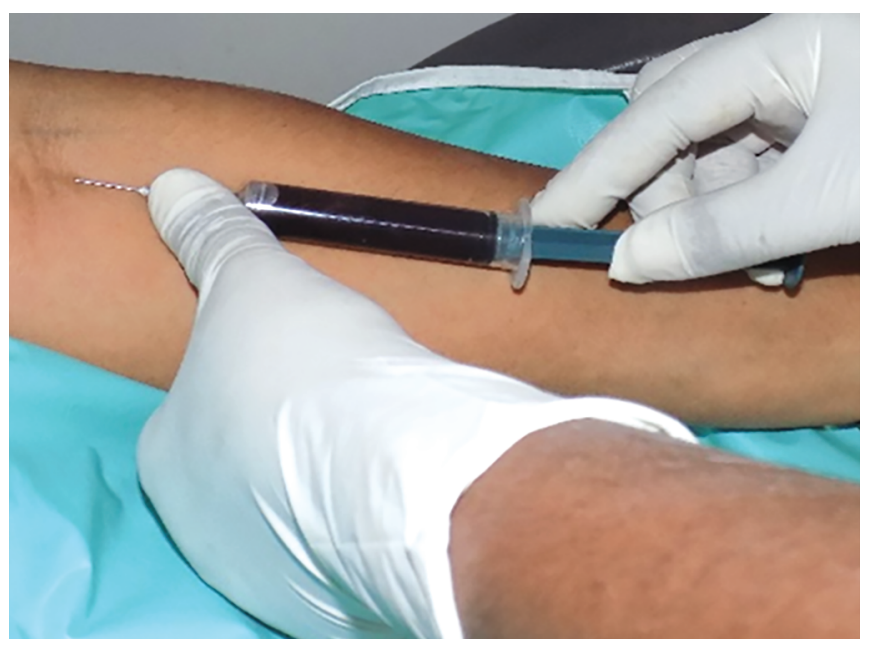

Fig. 1: A total of $5 \mathrm{~mL}$ collection of blood sample 


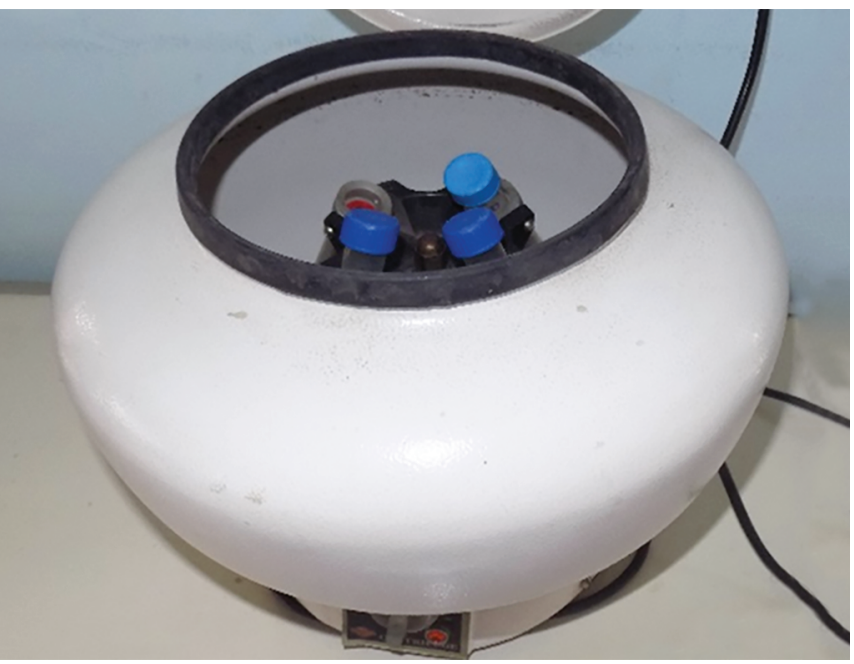

Fig. 2: Centrifugation of blood sample

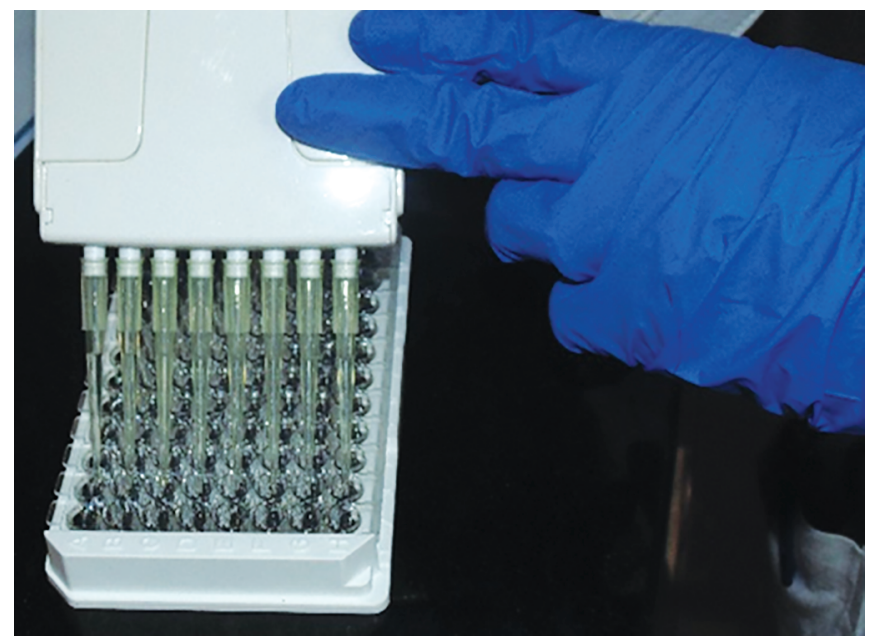

Fig. 4: Cytokine level was determined using ELISA kit

baseline and 4 weeks post therapy in the study group ( $p<0.05$; Table 2).

In the control group, there was a statistically significant difference in the periodontal and biochemical parameters at baseline and 4 weeks post therapy ( $p<0.05$; Table 3 ).

In the multiple comparison on serum level of TNF- $a$, both the groups showed a significant difference following nonsurgical periodontal therapy ( $p<0.05$; Table 4$)$.

In the multiple comparison on serum level of IL- 6 , both the groups showed a significant difference following nonsurgical periodontal therapy $(p<0.05$; Table 5$)$.

\section{Discussion}

Periodontal diseases occur due to a complex interplay of bacterial infection and host response, often modified by behavioral factors and various systemic conditions. ${ }^{14}$ In recent years, there has been a shift in interest from understanding periodontal manifestations of systemic diseases to linking the role of periodontal infections with various systemic diseases. ${ }^{15}$ The associations between periodontal diseases and systemic diseases, especially those causing hormonal disturbances such as diabetes mellitus and thyroid dysfunction had received great attention in periodontal literature in recent years.

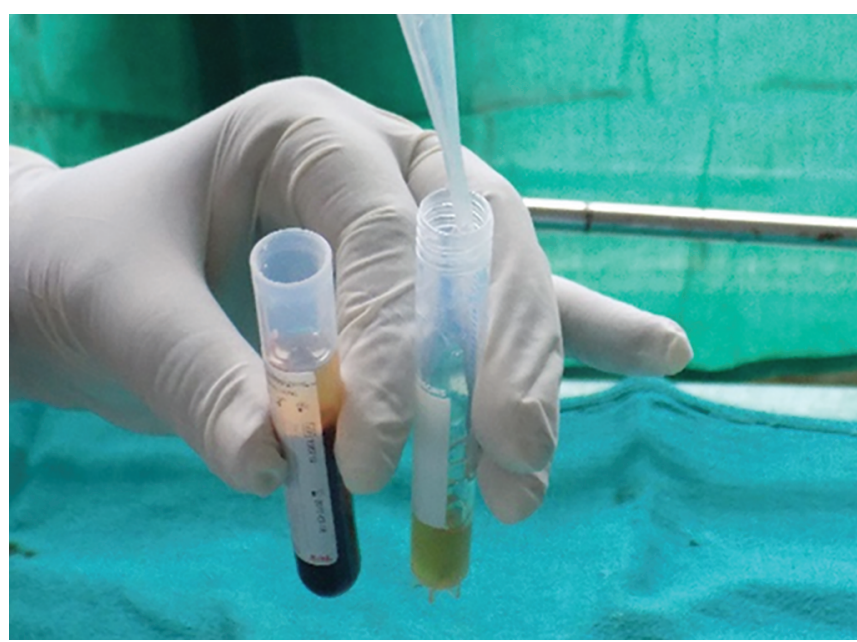

Fig. 3: Serum trasferred to vial

Many in vivo models demonstrated that the role of cytokines in pathophysiology had demonstrated that a given inflammatory stimulus results not in the generation of a single cytokine but rather a complex cascade of cytokine release. ${ }^{16}$ In hyperthyroidism, there will be an increased level of IL- 6 which will have an stimulatory effect on osteoclast differentiation and proliferation, with bone resorption as final effect. ${ }^{17}$ This associates the relationship between periodontitis and hyperthyroidism.

In the present study, a total of 40 subjects were selected and categorized into two groups, each group comprising of 20 subjects-group I (control group: systemically healthy subject with chronic periodontitis) and group II (study group: subjects with chronic periodontitis and hypothyroidism). Hypothyroidism is a clinical disease state occurring when there is insufficient thyroid hormone available to the target tissues. Serum TSH concentrations represent the most reliable indicators of thyroid status.

Several studies assessed the potential association between hypothyroidism and periodontitis. In the present study, at baseline, the periodontal parameters like plaque index, gingival index, probing pocket depth, and clinical attachment level were higher in group I (control group); the periodontal parameters were higher with mean values of $1.19 \pm 0.20,1.44 \pm 0.31,3.26 \pm 0.35$, and $3.67 \pm$ 0.5 , respectively. Similarly, in group II (study group), the periodontal parameters were higher at baseline: $1.19 \pm 0.22,1.47 \pm 0.23,3.36 \pm$ 0.21 , and $3.51 \pm 0.26$, respectively. This was in support of a similar study conducted by Babu and Patel where clinical parameters such as plaque index and gingival index were significantly higher in the study group (thyroid dysfunction) than the control healthy subjects. ${ }^{18}$ In a study conducted by Hanau et al., the clinical parameters were higher in study group (hypothyroid) which was similar to a study conducted by Babu and Patel et al. ${ }^{19}$ In our study, there was no significant difference for the periodontal parameters between group I and group II at baseline. Kothiwale et al. in a case report reported the influence of thyroid hormone dysfunction and its impact on periodontal disease progression, systemic health of the patient, and the management of periodontal disease. ${ }^{20}$

In the present study, we measured serum IL- 6 and TNF- $a$ by ELISA sandwich method. In this study, IL- 6 was selected because TNF- $a$ has been demonstrated to be an important stimulus for IL- 6 production. ${ }^{21}$ TNF- $a$ is a polypeptide with a multitude of effects. It plays a major role in the immunological cascade leading to 
Nonsurgical Periodontal Therapy and Hypothyroidism

Table 1: Demographic characteristics of the study population

\begin{tabular}{lllllll}
\hline & Gender & & \multicolumn{4}{c}{ Number of subjects in different age groups } \\
\cline { 2 - 2 } \cline { 5 - 7 } Groups & Female & & 18 -30 years & $31-40$ years & $41-50$ years & $51-60$ years \\
\hline Group I & 20 & 3 & 7 & 7 & 6 & 3 \\
Group II & 20 & 4 & 8 & 2 & 2 \\
\hline
\end{tabular}

Table 2: Comparison of mean periodontal and biochemical parameters values within the study group

\begin{tabular}{llll}
\hline & \multicolumn{2}{c}{ Study group (mean \pm SD) } & \\
\cline { 2 - 3 } Observations & Pretreatment & Posttreatment & p value \\
\cline { 1 - 3 } Plaque index & $1.19 \pm 0.22$ & $0.07 \pm 0.0$ & $0.001^{*}$ \\
Gingival index & $1.47 \pm 0.23$ & $0.52 \pm 0.70$ & $0.001^{*}$ \\
$\begin{array}{l}\text { Probing pocket } \\
\text { depth }(\mathrm{mm})\end{array}$ & $3.36 \pm 0.21$ & $2.35 \pm 0.21$ & $0.004^{*}$ \\
$\begin{array}{l}\text { Clinical attachment } \\
\text { level }(\mathrm{mm})\end{array}$ & $3.51 \pm 0.26$ & $2.42 \pm 0.21$ & $0.001^{*}$ \\
TNF-a $(\mathrm{pg} / \mathrm{mL})$ & $0.18 \pm 0.27$ & $0.07 \pm 0.03$ & $0.001^{*}$ \\
IL-6 $(\mathrm{pg} / \mathrm{mL})$ & $0.31 \pm 0.11$ & $0.13 \pm 0.06$ & $0.001^{*}$ \\
\hline
\end{tabular}

${ }^{*} p<0.05$ significant compared pre- and posttreatment within the study group $\mathrm{SD}$, standard deviation

Table 3: Comparison of mean periodontal and biochemical parameters within the control group

\begin{tabular}{llll}
\hline & \multicolumn{2}{c}{ Control group (mean \pm SD) } & \\
\cline { 2 - 3 } Observations & Pretreatment & Posttreatment & p value \\
\hline Plaque index & $1.19 \pm 0.20$ & $0.09 \pm 0.06$ & $0.001^{*}$ \\
Gingival index & $1.44 \pm 0.31$ & $0.49 \pm 0.78$ & $0.001^{*}$ \\
$\begin{array}{l}\text { Probing pocket depth } \\
(\mathrm{mm})\end{array}$ & $3.26 \pm 0.35$ & $2.31 \pm 0.28$ & $0.001^{*}$ \\
$\begin{array}{l}\text { Clinical attachment } \\
\text { level }(\mathrm{mm})\end{array}$ & $3.67 \pm 0.55$ & $2.50 \pm 0.43$ & $0.001^{*}$ \\
TNF-a $(\mathrm{pg} / \mathrm{mL})$ & $0.16 \pm 0.01$ & $0.10 \pm 0.04$ & $0.001^{*}$ \\
IL-6 $(\mathrm{pg} / \mathrm{mL})$ & $0.19 \pm 0.05$ & $0.11 \pm 0.02$ & $0.001^{*}$ \\
\hline
\end{tabular}

${ }^{*} p<0.05$ significant compared pre- and posttreatment within the control group $\mathrm{SD}$, standard deviation

Table 4: Multiple comparisons of TNF-a between the groups

\begin{tabular}{lll}
\hline Groups & TNF-a (mean $\pm S D)(p g / m L)$ & $p$ value \\
\hline Study pretreatment & $0.18 \pm 0.27$ & \\
Study posttreatment & $0.07 \pm 0.03$ & $0.001^{*}$ \\
Control pretreatment & $0.16 \pm 0.01$ & $0.001^{\#}$ \\
Control posttreatment & $0.10 \pm 0.04$ & $0.001^{*, \$}$
\end{tabular}

${ }^{*} p<0.05$ significant compared study group pretreatment with other groups

${ }^{\#} p<0.05$ significant compared study group posttreatment with other groups

$\$_{p}<0.05$ significant compared control group pretreatment with other groups

$\mathrm{SD}$, standard deviation

inflammatory response and stimulating the release of a variety of other mediators including IL-2, IL-6, and platelet-activating factor. ${ }^{22}$

The results of our study showed a higher value in the serum levels of TNF-a in group I (control group) and group II (study group) at baseline with mean values of $0.16 \pm 0.001$ and $0.18 \pm 0.27$, respectively (Tables 3 and 4). Similarly, higher value in the serum
Table 5: Multiple comparisons of IL-6 between the groups

\begin{tabular}{lll}
\hline Groups & $I L-6(\mathrm{pg} / \mathrm{mL})($ mean $\pm S D)$ & $p$ value \\
\hline Study pretreatment & $0.31 \pm 0.11$ & \\
Study posttreatment & $0.13 \pm 0.06$ & $0.001^{*}$ \\
Control pretreatment & $0.19 \pm 0.05$ & $0.001^{*, \#}$ \\
Control posttreatment & $0.11 \pm 0.02$ & $0.001^{*, \$}$ \\
\hline
\end{tabular}

${ }^{*} p<0.05$ significant compared study group pretreatment with other groups

${ }^{\#} p<0.05$ significant compared study group posttreatment with other groups

$s_{p}<0.05$ significant compared control group pretreatment with other groups

$\mathrm{SD}$, standard deviation

levels of IL-6 in group I (control group) and group II (study group) with mean values of $0.19 \pm 0.05$ and $0.31 \pm 0.11$ was observed at baseline, respectively (Table 5). This was similar with a study conducted by Adriana Monea et al. where mean values of serum TNF- $\alpha$ and IL- 6 for patients with hypothyroidism were higher than healthy subjects. ${ }^{23}$ The mean values of serum IL- 6 and TNF-a between group I and group II were not statistically significant. To support this, as for now no study had compared serum TNF-a and IL- 6 between subjects with hypothyroidism and systemically healthy subjects with chronic periodontitis.

Our study was an interventional study in which all the clinical parameters and serum IL- 6 and TNF- $\alpha$ levels were reevaluated after 4 weeks of nonsurgical periodontal therapy in both the groups, and results showed a significant reduction in all the clinical parameters in both the groups. This was similar with a previous study conducted by Goutoudi et al. where periodontal treatment showed improvement in all clinical parameters in chronic periodontitis patients. ${ }^{24}$ But the differences in the clinical parameters following nonsurgical periodontal therapy were not statistically significant between study and control groups. The possible underlying mechanism for the insignificance may be due to the influence of hypothyroid medication and nonsurgical periodontal therapy in the study group (group II).

The serum TNF-a levels in both the groups after nonsurgical periodontal therapy showed a significant decline from its baseline values. We observed a statistically significant reduction of TNF-a level in group I and group II from its baseline. However, there is no evidence to support the influence of nonsurgical periodontal therapy in lowering the serum level of TNF-a in chronic periodontitis with or without hypothyroidism.

The serum IL- 6 in both the groups after nonsurgical periodontal therapy showed a significant reduction from its baseline (Tables 2 and 3). This was in accordance with the study conducted by D'Aiuto et al. where after delivery of standard nonsurgical periodontal therapy, a significant reduction in serum IL- 6 was observed in chronic periodontitis patients. ${ }^{25}$ However, there is no evidence to support the influence of nonsurgical periodontal therapy in the serum level of IL- 6 in chronic periodontitis patients with hypothyroidism. 
The mean value of serum IL- 6 and TNF- $a$ between group I and group II after nonsurgical periodontal therapy was not statistically significant. The probable reason for the insignificance may be due to the reduced microbial load and thereby controlling inflammatory process following nonsurgical periodontal therapy. ${ }^{26}$ It is well established that the endocrine system can modulate the immune system in a bidirectional manner. ${ }^{27}$ Indeed, the relationship between diabetes mellitus and periodontitis offers a potential explanation for how acute and chronic inflammation, such as what occurs during periodontitis, might affect thyroid hormone production. ${ }^{28}$

The patients with long-standing hypothyroidism may have increased subcutaneous mucopolysaccharides. This may decrease the ability of small blood vessels to constrict when cut and may result in increased bleeding. ${ }^{29}$ This may be a challenge during periodontal surgical procedures.

In patients with diabetes mellitus, meta-analysis indicate that $\mathrm{HbA} 1 \mathrm{c}$ reduction of around $0.4 \%$ can be anticipated following effective nonsurgical periodontal therapy. The mechanism by which this occurs are not yet clear, but probably relate to reduced systemic inflammation following the treatment and resolution of periodontal inflammation. ${ }^{30}$ Till date, there are only limited data concerning the influence of scaling and root planing in chronic periodontitis patients with hypothyroidism.

However, to the best of our knowledge, this is the first study that evaluated clinical parameters and biochemical parameters following the nonsurgical periodontal therapy on chronic periodontitis patients with hypothyroidism. Based on these results, following nonsurgical periodontal therapy, serum levels of IL- 6 and TNF-a were reduced in systemically healthy subjects with chronic periodontitis and chronic periodontitis patients with hypothyroidism. This study provides evidence that nonsurgical periodontal therapy contributes to a reduction in serum levels of IL- 6 and TNF- $\alpha$ in these patients. Larger controlled trials are needed to confirm these findings.

The drawback of this study includes (1) age group included in the study ranged from 18 to 60 years, (2) sample size selected for the study was small, and (3) the study group with no hypothyroid medication would be desirable to observe the effects of periodontal therapy on serum cytokine levels and biochemical parameters. Hence, by overcoming these drawbacks, a significant result could be achieved in the future studies.

\section{CONCLUSION}

Serum levels of IL-6 and TNF-a were reduced following nonsurgical periodontal therapy in systemically healthy subjects with chronic periodontitis and chronic periodontitis patients with hypothyroidism. This study provides evidence that nonsurgical periodontal therapy contributes to a reduction in serum levels of IL- 6 and TNF- $\alpha$ in these patients. Larger controlled trials are needed to confirm these findings.

\section{References}

1. Gaspersic R, Stiblar-Martincic D, Osredkar J, et al. Influence of subcutaneous administration of recombinant TNF- $a$ on ligatureinduced periodontitis in rats. J Periodontal Res 2003;38(2):198-203. DOI: 10.1034/j.1600-0765.2003.01395.x.

2. Klein I, Ojamaa K. Thyroid hormone and the cardiovascular system. New England J Med 2001;344(7):501-509. DOI: 10.1056/ NEJM200102153440707.
3. Akalin A, Colak O, Alatas $\mathrm{O}$, et al. Bone remodelling markers and serum cytokines in patients with hyperthyroidism. Clin Endocrinol (Oxf) 2002;57(1):125-129. DOI: 10.1046/j.1365-2265.2002.01578.x.

4. Al-Rubbaey YA. Oral health status and dental treatment needs in relation to salivary constituents and parameters among a group of patients with thyroid dysfunction. J Baghdad Colle Dent 2010;22(1):105-108.

5. Hodkinson $\mathrm{CF}$, Simpson EE, Beattie JH, et al. Preliminary evidence of immune function modulation by thyroid hormones in healthy men and women aged 55-70 years. J Endocrinol 2009;202(1):55. DOI: 10.1677/JOE-08-0488.

6. Liu YC, Lerner UH, Teng YT. Cytokine responses against periodontal infection: protective and destructive roles. Periodontol 2000 2010;52(1):163-206. DOI: 10.1111/j.1600-0757.2009.00321.x.

7. Sekeroglu MR, Altun ZB, Algün E, et al. Serum cytokines and bone metabolism in patients with thyroid dysfunction. Adv Ther 2006;23(3):475-480. DOI: 10.1007/bf02850169.

8. McGee JM, Tucci MA, Edmundson TP, et al. The relationship between concentrations of proinflammatory cytokines within gingiva and the adjacent sulcular depth. J Periodontol 1998;69(8):865-871. DOI: 10.1902/jop.1998.69.8.865.

9. Kanatani $M$, Sugimoto $T$, Sowa $H$, et al. Thyroid hormone stimulates osteoclast differentiation by a mechanism independent of RANKLRANK interaction. J Cell Physiol 2004;201(1):17-25. DOI: 10.1002/ jcp.20041.

10. Vestergaard P,Mosekilde L. Fractures in patients with hyperthyroidism and hypothyroidism: a nationwide follow-up study in 16,249 patients. Thyroid 2002;12(5):411-419. DOI: 10.1089/105072502760043503.

11. Aziz AS, Kalekar MG, Benjamin T, et al. Effect of nonsurgical periodontal therapy on some oxidative stress markers in patients with chronic periodontitis: a biochemical study. World J Dent 2013;4(1):17-23. DOI: 10.5005/jp-journals-10015-1196.

12. Unnikrishnan AG, Menon UV. Thyroid disorders in India: an epidemiological perspective. Indian J Endocrinol Metab 2011;15(2):S78. DOI: 10.4103/2230-8210.83329.

13. Baskin HJ, Cobin RH, Duick DS, et al. American association of clinical endocrinologists medical guidelines for clinical practice for the evaluation and treatment of hyperthyroidism and hypothyroidism: AACE thyroid task force. Endocrine practice 2002;8(6):457-469. DOI: 10.4158/1934-2403-8.6.457.

14. Kim J, Amar S. Periodontal disease and systemic conditions: a bidirectional relationship. Odontology 2006;94(1):10-21. DOI: 10.1007/s10266-006-0060-6.

15. Aimetti M, Romano F, Nessi F. Microbiologic analysis of periodontal pockets and carotid atheromatous plaques in advanced chronic periodontitis patients. J Periodontol 2007;78(9):1718-1723. DOI: 10.1902/jop.2007.060473.

16. Creasey AA, Stevens P, Kenney J, et al. Endotoxin and cytokine profile in plasma of baboons challenged with lethal and sublethal Echerichia coli. Circ Shock 1991;33(2):84-91.

17. Fenkci S, Corapcioglu D, Erdogan G. The effects of thyrotoxicosis on serum IL-6 and bone turnover in premenopausal women. Turkish J Endocrinol Metabolism 2001;3:97-101.

18. Babu NV, Patel PB. Oral health status of children suffering from thyroid disorders. J Indian Soc Pedodont Prevent Dent 2016;34(2):139. DOI: 10.4103/0970-4388.180443.

19. Hanau KJ, Razzoqi Naoom E, Mahammed HO. CPITN in Iraqi females with thyroid dysfunction. Mustansir Dent J 2018;9(1):99-106.

20. Kothiwale S, Panjwani V. Impact of thyroid hormone dysfunction on periodontal disease. J Scienti Soc 2016;43(1):34. DOI: 10.4103/09745009.175456.

21. Jirik FR, Podor TJ, Hirano T, et al. Bacterial lipopolysaccharide and inflammatory mediators augment IL- 6 secretion by human endothelial cells. J Immunol 1989;142(1):144-147.

22. Bonavida B, Paubert-Braquet $M$, Hosford $D$, et al. The involvement of platelet-activating factor (PAF)-induced monocyte activation and tumor necrosis factor (TNF) production in shock. Prog Clin Biol Res 1989;308:485-489. 
23. Monea A, Elod N, Sitaru A, et al. Can thyroid dysfunction induce periodontal disease? Euro Scienti J 2014;10(15):1857-7431.

24. Goutoudi P, Diza E, Arvanitidou M. Effect of periodontal therapy on crevicular fluid interleukin- 6 and interleukin-8 levels in chronic periodontitis. Int J Dent 2012;2012 10.1155/2012/362905.

25. D'Aiuto F, Parkar M, Andreou G, et al. Periodontitis and systemic inflammation: control of the local infection is associated with a reduction in serum inflammatory markers. J Dent Res 2004;83(2): 156-160. DOI: $10.1177 / 154405910408300214$.

26. Pawlowski AP, Chen A, Hacker BM, et al. Clinical effects of scaling and root planing on untreated teeth. J Clin Periodontol 2005;32(1):21-28. DOI: 10.1111/j.1600-051X.2004.00626.x.
27. Klein JR, Maywood NJ. The immune system as a regulator of thyroid hormone activity. Exp Biol Med 2006;231(3):229-236. DOI: 10.1177/153537020623100301.

28. Borgnakke WS, Ylostalo PV, Taylor GW, et al. Effect of periodontal disease on diabetes: systematic review of epidemiologic observational evidence. J Periodontol 2013;84(4):S135-S152. DOI: 10.1902/jop.2013.1340013.

29. Silverston SF. Endocrine disease. In: Greenberg MS, Glick M. Burket's Oral Medicine diagnosis and treatment. 10th ed., Hamilton, Ontario: BC Decker Inc; 2003. pp. 578-591.

30. Simpson TC, Needleman I, Wid SH, et al. Cochrane Database Syst Rev 2010(5):CD004714. DOI: 10.1002/14651858.CD004714.pub2. 